Chapter 6

\title{
A Framework for the Systematic Characterization of the Aortic Valve Complex by Real-Time Three Dimensional Echocardiography: Implications for Transcatheter Aortic Valve Replacement
}

\author{
Daniel Zalkind, Michael Kim and Ernesto E. Salcedo \\ Additional information is available at the end of the chapter \\ http://dx.doi.org/10.5772/56021
}

\section{Introduction}

Transcatheter aortic valve replacement (TAVR) has become a viable alternative to surgical aortic valve replacement in both high-risk and inoperable patients with symptomatic severe aortic stenosis [1]. A successful TAVR procedure is heavily dependent on appropriate patient selection and detailed morphologic characterization of the aortic valve complex to prevent device migration, coronary artery compromise and paravalvular leak. Two-dimensional echocardiography (2DE) and Doppler methods have traditionally been used to characterize the presence and severity of aortic stenosis [2]; however the detailed structural analysis of the aortic valve complex required for successful TAVR demands a more precise analysis of not only the aortic leaflets, but of the left ventricular outflow tract dimensions, aortic valve annulus, and the aortic root. Computed tomography (CT) $[3,4,5,6]$ and two dimensional transesophageal echocardiography (2D TEE) [7] are traditionally used to obtain this information. Although transthoracic and transesophageal three-dimensional echocardiography (3D Echo) provide detailed characterization of the aortic valve complex, these advanced modalities are not currently being routinely used in the evaluation of patients considered for TAVR. The purpose of this chapter is to provide a framework for the 3D echocardiographic evaluation of the aortic valve complex and its implications for TAVR.

\subsection{The aortic valve complex}

The aortic valve complex includes the aortic valve leaflets and surrounding structures defining a functional unit that allows, under normal circumstances, unidirectional and unobstructed 
blood flow to exit the left ventricle and to enter the ascending aorta. The clinical anatomy of the aortic root has been well characterized in other publications [8,9] and is summarized below. More recently, the anatomy of the aortic valve complex and its implications in TAVR have been elegantly reviewed by Piazza [10]. The components of the aortic valve complex are illustrated in (Figure 1).

\section{Anatomy and clinical application}

\subsection{General anatomical landmarks}

As described above, the aortic annulus is one part of the aortic valve complex that is comprised of the muscular ventricular LVOT, the aortic valve, aortic leaflets, sinuses of Valsalva, the sinotubular junction and the ascending aorta [11]. The aortic annulus diameter and general geometry, which is crucial for pre-procedural planning, valve implantation and post-procedural follow-up can be considered to be a virtual ring joining the basal attachments of all three aortic valve cusps, representing the inlet from the left ventricular outflow tract into the aortic root [12]. The annulus described by surgeons is usually the semilunar crown-like structure demarcated by the hinges of the leaflets. There is also the true ring, comprised of the sinotubular junction, demarcated by the sinus ridge and the related sites of attachment of the peripheral zones of apposition between the aortic valve leaflets. This forms the outlet of the aortic root into the ascending aorta.

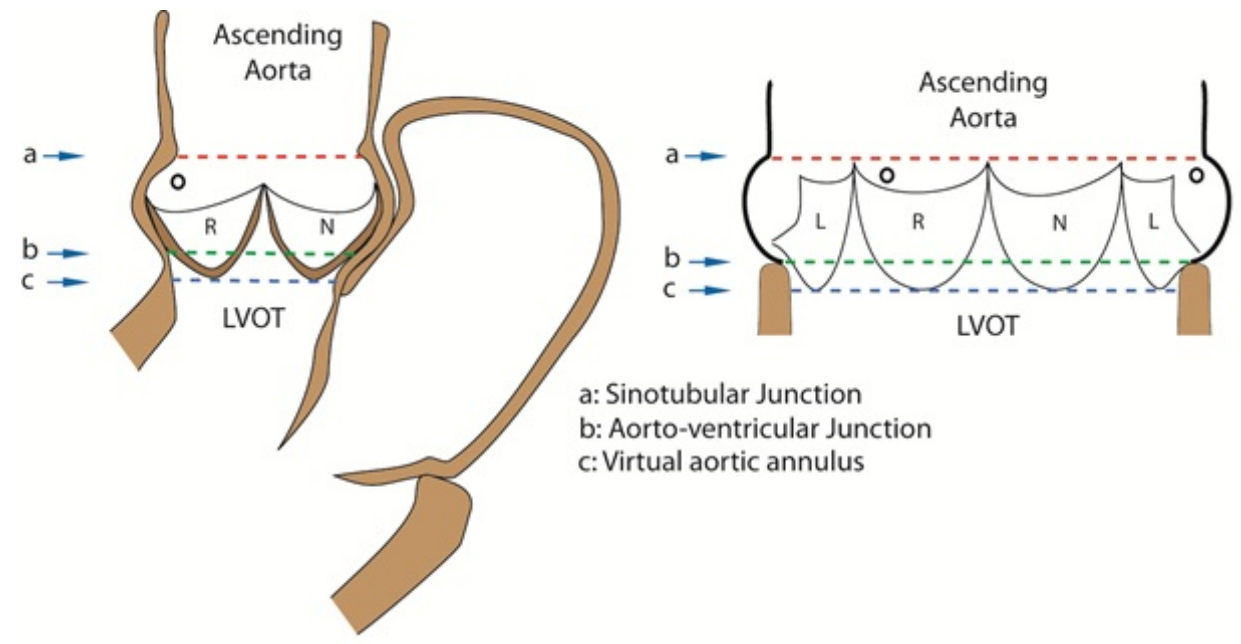

Figure 1. Aortic valve complex with virtual and anatomical rings.

The larger part of the noncoronary leaflet of the valve, along with part of the left coronary leaflet, is in fibrous continuity with the aortic or anterior leaflet of the mitral valve, 
with the ends of this area of fibrous continuity being thickened to form the so-called fibrous trigones. It is these trigones that anchor the aortic-mitral valvular unit to the roof of the left ventricle. The valvular complex is a dynamic structure, with the geometric parameters changing continuously during the phases of the cardiac cycle and in relation to changes in pressure within the aortic root. From diastole to systole, the change in the diameter at the level of the outlet and at the base of the valve has been noted to increase more than $10 \%$, according to several earlier publications. Proper functioning of the native aortic valve depends on the proper relationship between the leaflets within the aortic root. Not only do variations exist among individuals in the dimensions of the root, but in the same individual, marked variations can exist in all aspects of the dimensions of the individual leaflets, including the height, width, surface area, and volume of each of the supporting sinuses of Valsalva.

\subsection{Application of echocardiography}

Transthoracic echocardiography (TTE) is a critical portion of patient selection for TAVR and complements the clinical criteria for TAVR due to its robust ability to provide both anatomical and hemodynamic data. According to the latest European Association of Echocardiography and the ASE 2011 consensus document [13], the role of TTE is to specifically assess the annular dimension and detailed anatomic characteristics of the aortic valve, including the number, mobility, and thickness of cusps, as well as the extent and distribution of calcification.

Patients being considered for TAVR should undergo a pre-TAVR 2D/Doppler TTE evaluation to serve as a baseline for post-TAVR comparison. In addition 3D TTE should be performed to more precisely delineate the aortic valve complex in order to better determine patient suitability for TAVR. Transthoracic echo plays a key role in establishing the presence of severe AS with Doppler assessment of peak and mean transaortic gradients as well as AVA calculation by the continuity equation. Per the current ACC/AHA guidelines, severe AS is defined by an AVA of $<1 \mathrm{~cm} 2(<0.6 \mathrm{~cm} 2 / \mathrm{m} 2)$ or a mean aortic valve gradient of $>40 \mathrm{mmHg}$. The aortic valvular dimension must be assessed with echocardiography, as the current guidelines recommend a $23 \mathrm{~mm}$ prosthesis for transverse aortic annular diameters of 18-21 $\mathrm{mm}$ (measured at the level of aortic cusp insertion) and a $26 \mathrm{~mm}$ prosthesis for aortic annular diameters of $22-25 \mathrm{~mm}$. There is also a larger $29 \mathrm{~mm}$ SAPIEN valve which is currently available outside of the United States which can accommodate 26-29mm annular diameters.

Using TTE, assessing the annular dimension and detailed anatomic characteristics of the aortic valve, including the number, mobility, and thickness of cusps, as well as the extent and distribution of calcification should be described. LV and right ventricular dimensions and function, aortic regurgitation, and the structure and function of the other valves should be evaluated. Other valvular disease such as severity of valvular regurgitation or presence of bioprothetic valves in other positions must also be closely evaluated as such findings may preclude TAVR. Left ventricular thrombus and valvular vegetations must be ruled out due to the risk of embolization during sheath manipulation prior to or during deployment. LVOT obstruction and calcification should also be evaluated as it can interfere with device deployment and can lead to migration of the valve. Pericardial fibrosis or pericardial patch should 
be evaluated with either echocardiography or CT as the transapical approach requires a thoracotomy and pericardioctemy.

During the implantation of a transcatheter aortic valve, contact between the valve struts or between the mechanically dilated and moved native calcifications can skirt the membranous septum, potentially instigating high-degree atrioventricular heart block caused by the compression of the left bundle. This phenomenon is of particular concern in patients with preexisting right bundle branch block (RBBB).

Currently, bicuspid aortic valve is an exclusion criterion for TAVR because an elliptical valvular orifice may predispose to an increased risk of incomplete and incorrect deployment of the aortic prosthesis, predisposing to a risk of paravalvular leak or valve embolization post implantation. Moreover, the risk of aortic complications in patients with bicuspid aortic valves, such as spontaneous aortic dissection, may be increased due to abnormal arterial wall structure.

\subsection{Aortic and mitral valve interaction}

Although difficult to study and to characterize with traditional 2D echocardiography, the interaction between the mitral and aortic valves can vary in intensity and may have subtle effects on the success or failure of TAVR. A study of this interaction from Veronesi and colleagues demonstrated that the interaction between the mitral annulus surface area and aortic annulus projected area changed reciprocally over time due to the physical interaction through this fibrous continuity [14]. The aortic annulus projected area increased $17 \%$ from end diastole to early systole and decreased to a minimum during isovolumic relaxation. The angle between the mitral and aortic annuli decreased by $5 \%$ from diastole to systole. It is reasonable to infer that valve undersizing, resulting in more frequent and/or severe perivalvular aortic regurgitation, may result if the coupling between the aortic and mitral valves is not appreciated. Other work in mitral-aortic valve coupling was presented at the ACC conference in 2012 and described that aortic stenosis patients have reduced mitral annular size and displacement. After TAVR, mitral annular size remains reduced due to the presence of the calcified aortic valve, which is compressed along the aortic root by the TAVR valve struts. This may also contribute to the increase in aortic-mitral angle post-TAVI [15].

\subsection{General Description of the Sinuses of Valsalva and coronary artery ostia}

The aortic root is a geometrically complicated structure that includes the sinuses of Valsalva which are in direct communication with the coronary cusp insertion points. The coronary artery ostia are in the sinuses and thus give name to the sinuses (left, right and non-coronary) [16]. The height of the sinuses and the proximity to the aortic valve cusps in systole are two important parameters to consider in the pre-TAVR planning

For pre-TAVR planning, the LVOT view (110-140 degrees of omni-plane) should be used to visualize the height of the sinus, defined as the distance from the aortic annulus to the sinotubular junction (tapering of the sinuses to the ascending aorta diameter) [17]. We have found success in visualizing the sinuses of Valsalva with transthoracic 3D by using multi-plane 
reconstruction (MPR) [18] and measuring the longest diameter and area. MPR can also be used to assess the height of the sinuses, best visualized with our technique from the "Full Volume" acquisition from the parasternal long window as shown in figures 2-4. Alternatively, CT imaging can also be used with a similar MPR technique.

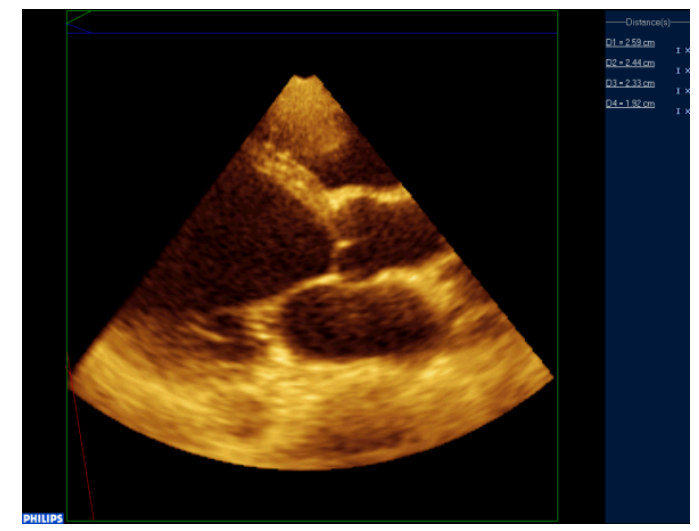

Figure 2. LVOT view from parasternal long MPR

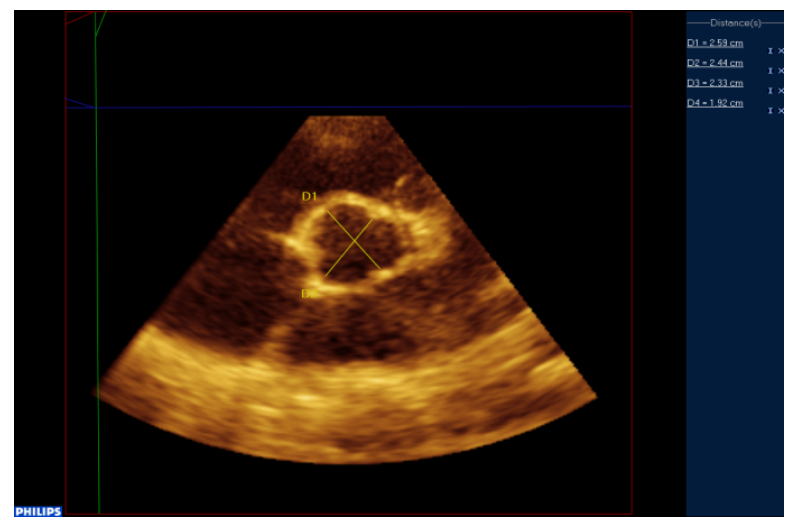

Figure 3. Aortic annular dimension from parasternal long MPR

\subsection{Fibrous interleaflet triangles}

The portion of the aortic wall that is beneath the apices formed by the attachment of the aortic leaflets straddles the ventricular and aortic sides of the aortic valve. This region of the heart is exposed to the ventricular hemodynamic forces. Also known as trigones, these structures help to support the integrity of the aortic valve [19]. Some authors have made the analogy to a suspension bridge where the collagenous thickening at the zone of coaptation and the hinges 

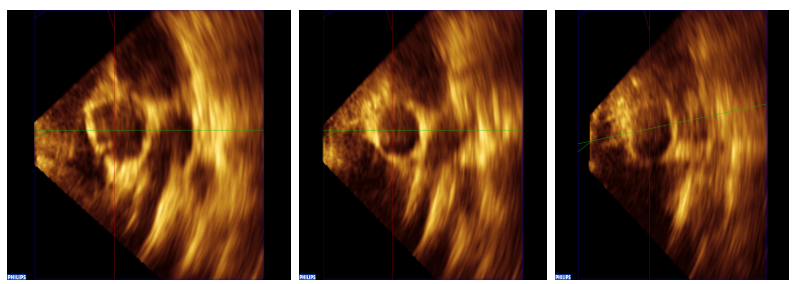

Figure 4. Sinus of valsva (left), sinotubular junction (center) and ascending aorta (right). MPR from parasternal long view.

of the leaflets correspond to support cables. These interleaflet triangles are crucial for proper valvular function. When one or more of them are vestigial, or very small, the valve can become stenotic due to a change in the geometry. It is challenging to view these structures with transthoracic echocardiography but TEE can sometime be used to identify them if the valvular motion is slowed down during the post-processing stage and they are identified in mid systole or possibly mid diastole, depending on the plane of imaging that is used. Three-dimensional echocardiography does not quite have the resolution to discriminate between these thin structures and the valve leaflets. These triangles are currently not part of the TAVR planning protocols at most institutions at this time.

\subsection{Tubular portion of ascending aorta}

Aortic valvular disease such as aortic stenosis and aortic regurgitation can often be accompanied by ascending aortic dilatation. In a recent, large case series from Baylor Medical Center, of the 1385 patients who had isolated aortic valve replacement, 59 also had aortic root resection [20]. The largest ascending aortic diameter (multiple imaging modalities were included) ranged from 4.5 to $6.7 \mathrm{~cm}$ (mean, $5.3+-0.5 \mathrm{~cm}$ ): these diameters were larger in the men than in the women (mean, $5.4 \mathrm{~cm}$ versus mean, $4.9 \mathrm{~cm}$ ). Although the association between bicuspid valve and aortopathy is well known, occasionally patient with severe AS may be referred for TAVR and the valve is so heavily calcified that the diagnosis of bicuspid valve (and thus their ineligibility for TAVR) is not known. Wide-sector acquisition in the left parasternal long axis can be used to visualize the ascending aorta and thus help to identify dilatation of the ascending aorta that may, in itself, warrant surgical correction. Alternatively, CT with contrast can be used to assess the ascending aorta.

\subsection{Aorto-mitral fibrosa}

The aorto-mitral fibrosa is usually a thin piece of fibrous tissue that lies between the mitral and aortic annuli. Best visualized in the parasternal long view on TTE, the fibrosa is usually thin [21]. Alternations to the geometry of the left ventricular inflow and outflow regions can lead to variations in the length of this fibrosa, such as in transposition of the great vessels, endocarditis or heavy calcification of either or both annuli. From parasternal short axis window, acquisition can be performed in "Live 3D Zoom" modality to examine the left ventricular outflow tract and mitral valve region. More detailed pictures can, of course, be obtained with TEE as well. 


\subsection{Pathology including subvalvular stenosis and HCM}

Echocardiography has been used to assess all of the structures mentioned above as well as hypertrophic cardiomyopathy (HCM) with substantial validation. In a thorough study of 77 pediatric patients with subaortic stenosis from the University of Michigan from 1983 to 1991, echocardiography was used to evaluate patients before and after surgical repair as well as follow them longitudinally as their gradients increased. In the 36 patients who underwent surgery, the pre-operative LVOT gradient decreased from 63 to $14 \mathrm{mmHg}$ [22]. TTE examinations were crucial in this patient population due to the complexity of the lesions. A discrete fibrous membrane or fibromuscular collar can frequently encircle the LVOT just beneath the aortic valve. Occasionally the long fibromuscular obstruction narrows the LVOT for several centimeters forming a tunnel. Accompanying aortic regurgitation can also be present and has been used as an indicator for urgent surgical correction. Three dimensional echocardiography can be especially useful in such cases as the aortic valvular pathology can be differentiated from the subaortic pathology and the aortic regurgitation can be fully described (eccentricity, vena contracta width and PISA) using 2D, 3D, pulsed doppler and color-flow doppler. Subaortic stenosis is a contraindication to CoreValve implantation. Additionally, the high incidence of concominant atrial and ventricular septal defects increases the utility of echocardiography as those defects can be readily visualized.

Hypertrophic cardiomyopathy (HCM) is the most common genetic cardiomyopathy with a prevalence of approximately $0.2 \%$. The clinical presentation is quite diverse but the sentinel findings of either concentric basal hypertrophy (with septal involvement), arrhythmias and sudden cardiac death can be recognized late in the disease process [23]. According to the 2011 American Society of Echocardiography guidelines for HCM, ventricular volumes, discrete wall thickness and LV geometry (apical vs. septal hypertrophy) can be defined. Dynamic obstruction of the LVOT during stress can be assessed but due to the need for continuous wave Doppler, can be confused with severe aortic stenosis. In the evaluation of patients with suspected concominant aortic stenosis and a dynamic LVOT obstruction from HCM, TEE imaging is crucial to separate the two pathologies. Additionally, papillary muscle hypertrophy/remodeling can also lead to the elongation of the mitral leaflet area and lead to further increases in the LVOT gradient due to the venture effect in the hypertrophied and/or hyperdynamic LVOT. These dynamic processes must be evaluated prior to any consideration of transcatheter treatment of aortic stenosis.

\section{Three dimensional echocardiography of the aortic valve complex}

\subsection{Background, physics and validation}

Two dimensional echocardiography has numerous limitations in assessing the volumes and geometry of complex cardiac structures such as the left ventricular cavity (for the purposes of LVEF assessment) as described above and include geometric assumptions, image plane positioning errors and imprecise endocardial boundary demarcation [24]. Over the last twenty years, numerous advances in ultrasound transducer technology and parallel computer 
processing has made the acquisition of 3D echocardiographic volumes possible using multiple formats (in the Philips Healthcare system but similar modes in the other manufacturers as well) including large sector ("Full Volume"), narrow sector format ("Live 3D"), wide sector focused ("Zoom 3D"), simultaneous biplane ("X-plane") modes. The different formats are provided because 3D echocardiography is a technically demanding technique with temporal and spatial resolution limitations. Each one of the formats or modes has strengths and weaknesses.

One of the first clinical studies documenting real-time 3D echocardiography was published in 1998. The phased-array volumetric 3D system was developed in the Duke University Center for Emerging Cardiovascular Technology and initially operated with 2.5- or 3.4-MHz transducers (both $14 \mathrm{~mm}$ in diameter) [25]. A 2D array and the pyramid-shaped volumetric scan composed of $43 \times 43$ square elements, each measuring $0.3 \times 0.3 \mathrm{~mm}$. The volume was scanned rapidly with 16-to-1 parallel processing in the receive system. Consequently, the overall scanned pyramidal volume was composed

of 256 small pyramids stacked side by side. Since then the transducer technology has increased substantially and current systems are solid state devices that operate at 5-7 $\mathrm{MHz}$ and are composed of more than 3000 array elements with the ability to acquire in the multiple modes above as well as to perform other advanced functions such as 3D color Doppler and color Mmode. This technology has been validated against nuclear magnetic resonance [26] and SPECT imaging [27].

Large sector acquisition ("Full Volume" on the Philips Healthcare system) is a technique for acquiring large volume sets such as the entire left ventricle over multiple cardiac cycles to allow the transducer/processor to sample small sectors of the entire volume during each cycle. The sectors are then "stitched" together by software algorithms into one large volume. This technique allows for large volumes to be imaged but relies on a regular heart rhythm that produces constant R-R intervals. Respiratory artifact can also be introduced if the ultrasound probe is affected leading to slightly different cardiac geometry to be sampled in the various sectors leading to "stitch" artifact. This technique has been used in our laboratory to define LV geometry as well to define the aortic annulus and the entirety of the aortic valve complex and proximal ascending aorta in one acquisition step. Apical and parasternal long positions are favored for this approach.

Narrow sector format ("Live 3D") is a technique which focuses on a narrow sector such as the aortic leaflets at the level of the Sinuses of Valsalva with high temporal resolution and frame rates above $30 \mathrm{~Hz}$ for visualization of rapidly moving structures such as fenestrations, vegetations or thrombi. Temporal resolution is now sufficient to visualize valvular motion as seen in figure 5 .

Wide sector focused ("Zoom 3D") allows for large acquisition fields that are relatively shallow which can be obtained anywhere along the long axis of the field of view. For example, the entire aortic valve annulus, leaflets and possibly the coronary ostia can be visualized. This approach is often coupled with the simultaneous orthogonal view format known as "X-plane". This $2 \mathrm{D}$ technique allows the sonographer to visualize complex structures in their traditional 

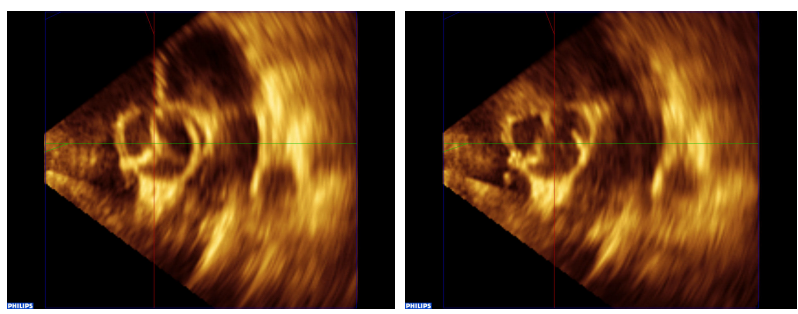

Figure 5. Aortic valve in diastole and systole with transthoracic 3D MPR reconstruction.

2D view and concurrently, in a split window format, visualize the orthogonal 2D plane. Especially useful in the identification of the coronary ostia and left atrial appendage thrombi and the aortic annular diameter.

\subsection{Role for 3D echo from EAE and ASE guidelines}

Since the development of the matrix-array transducer and real-time 3D echocardiography, the application to clinical practice has increased substantially due to improves accuracy and ease of use. Direct measurements of cardiac chambers without geometrical assumptions, noninvasive views of valvular structure and function as well as color Doppler assessment of regurgitation and stenosis are all improving and are gradually becoming well accepted and strongly recommended in the ASE guidelines [28].

Specifically 3D LV chamber quantification is now recommended as the best technique for evaluating LV end-diastolic and end-systolic volumes, leading to accurate quantification of LV ejection fraction. Several studies have now directly compared the accuracy of 3D measurements of $\mathrm{LV}$ volumes and $\mathrm{EF}$ have shown the superiority of the 3D approach over the 2D methodology, which was shown to consistently underestimate LV volumes.

Valvular heart disease has been studied as a possible application for RT3D as well. The most experience comes in mitral disease where the hyperbolic paraboloid ("saddle-shaped") geometry of the valve is better evaluated from multiple planes that are possible with 3D imaging. The current TAVR recommendations recommend that the aortic valve is imaged with $3 \mathrm{D}$ but the sizing of the valve still be performed with 2D echo in the LVOT view, usually accomplished at the mid-esophageal position at 120 degrees of omni tilt. LVOT measurements performed on the 3D dataset require multi-planar reconstruction (see below).

\subsection{Wide-sector acquisition and advances in post processing}

Although not currently endorsed for the pre-procedural evaluation for TAVR, transthoracic 3D imaging may have a special niche. Similar 3D capabilities as transesophageal echocardiography are available to the sonographer including RT3D with high-frame rate imaging (Live 3D) as well as breath-hold, large sector acquisition. The off-line processing software is currently aimed for LV ejection fraction quantification but, in the authors' experience, can be applied to the LVOT, aortic annulus and possibly the ascending aorta. 
Three-dimensional imaging requires optimal patient positioning to minimize the lung space between the thoracic wall and the myocardium (extreme left decubitus) along with minimal subcutaneous fat layer. For example we typically perform apical 3D imaging on dedicated echocardiography tables with fold-out windows to allow for proper transducer alignment with the long-axis of the LV. This is crucial for 3D imaging of the LVOT [29] as the focus is more on the imaginary line drawn through the proximal ascending aorta into the LV cavity as we have found that the acquisition volume needs to be positioned over the aortic valvular complex and the ascending aorta.

Often the increased calcium in the aortic annulus and on the leaflets causes shadowing of the aortic valve and ascending aorta. We have found that parasternal long 3D imaging with both Live3D and Full-Volume acquisition produces easily interpretable and physiologic images [30]. This view is crucial for highly calcified aortic valves or for HCM patient with substantially hypertrophied septum which can be calcified and can obstruct the aortic annulus.

Once the 3D sample volume is acquired in either the "Full Volume" or "Zoom 3D" formats, multi-plane reconstruction can be used to visualize the aortic valve complex in any plane. Most useful for the assessment of the aortic annular diameter as well as the diameter of the sinotubular junction, MPR allows the echocardiographer to solve the issue of "oblique" planes through round structures that can lead to under or overestimation of the annular diameter. In a recent retrospective analysis in the Journal of the American College of Cardiology, Wilson and colleagues suggested that CT imaging of the aortic annulus is more accurate and can lead to better sizing for TAVR, presumably leading to less perivalvular regurgitation (See below for the unique benefits of $3 \mathrm{D}$ echocardiography in perivalvular regurgitation).

An off-line processing feature on the Philips workstation known as "I-Slice" is an automatic software feature that isolates multiple segments of the LV and allows for separate evaluation. Mostly designed for the evaluation of LV function throughout different segments of the chamber, this feature can also be applied to the transthoracic evaluation of the aortic valve complex and the ascending aorta by applying it along the LVOT-to-ascending aorta axis. Orthogonal 2D slices at multiple levels of this area of interest can be displayed simultaneously and thus can help to identify calcium, maximal and minimal diameters and areas of supraaortic and sub-aortic stenosis without the need for multi-plane reconstruction (Fig. 6).

\section{Clinical application in aortic stenosis}

The publication of the PARTNER trial ushered in a new era in interventional cardiology and highlights the improved outcomes of TAVR vs. medical treatment. As originally reported by Braunwald and colleagues and recently further explored by Carabello and colleagues, aortic stenosis had a $2 \%$ incidence in the Cardiovascular Health Study and has a high mortality rate (especially with symptoms) when medical treatment alone is initiated. As per the 2008 EAE/ASE valvular guidelines, echocardiography has become the standard means for evaluation of aortic stenosis (AS) severity [31]. Cardiac catheterization 

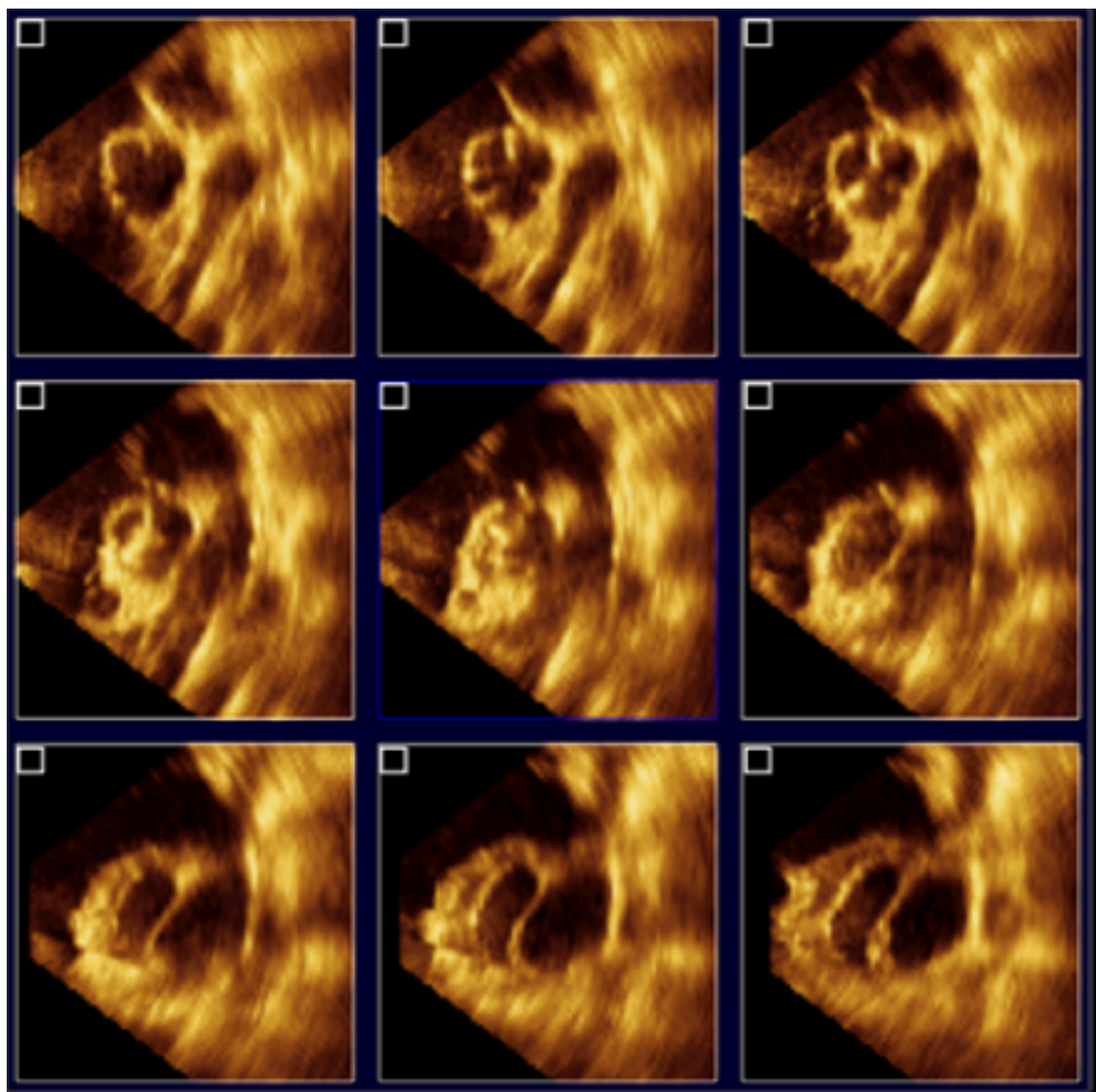

Figure 6. Philips "I-Slice" mode applied to the aortic valve complex.

is no longer recommended except in rare cases when echocardiography is non-diagnostic or discrepant with clinical data [32].

As mentioned above, non-invasive imaging including echocardiography is important and plays a central role in TAVR because accurate preoperative measurements of aortic annular sizes are crucial for selection of appropriate prosthesis sizes. One of the possible imaging modalities, 2D echocardiography underestimates aortic annular and LVOT dimensions compared when compared with multislice computed tomography (MSCT) and 3D echocardiography [33]. Three dimensional TEE has been promoted for more accurate diameter measurements. A study by $\mathrm{Ng}$ and colleagues, from the Netherlands, showed marked differences in the aortic annular dimension measurement between 2D TEE, 3D TEE circular annular assumption and 3D TEE with planimetry measurements based on overall valve area although all of the modalities were consistent when eccentricity was measured post TAVR (circular valve prosthesis). Using CT planimetered areas as "gold standard," 3D TEE plani- 
metered aortic annular and LVOT areas showed the least underestimation and narrowest limits of agreements compared with their respective calculated circular areas. After TAVR, both 3D TEE and CT demonstrated a decrease in the annular dimension and area due to the thickness of the valve stent thickness [34]. In contrast, the planimetered LVOT areas increased by both modalities due to a more circular geometry after TAVR.

Three dimensional TEE has another niche in aortic stenosis. Obstructions in the LVOT or postprocedural complications such as pannus formation in surgical valves can be visualized with real time 3D TEE [35]. A pannus that formed on a mitral valve that could be visualized only with 3D TEE was reported by Ozkan and colleagues in 2009. The same technology technique could be transferred to the aortic valve with the caveat that shadowing can often complicate visualization of intra-valvular pathology, based on our experience.

Finally, two dimensional echocardiography has recently been used to estimate effective orifice area after TAVR and the measurement of the LVOT diameter immediately proximal to the valve stent struts produced the most likely true effective orifice area with the minimal false negative prosthesis-patient-mismatch (AVA $<0.65 \mathrm{~cm} / \mathrm{m} 2)$ [36].

\subsection{Aortic insufficiency and perivalvular leaks}

Periprocedural complications of device deployment may limit the short-term and long-term results that have been reported. One common problem is perivalvular aortic regurgitation. Two dimensional $[37,38]$ and three dimensional echo has been validated in native valve regurgitation in previous studies $[39,40,41]$. Based on the previous experience in surgically-implanted valves, periprosthetic leak severity is defined as severe when greater than $25 \%$ of the ring circumference is involved by color Doppler [42]. While predictors for left ventricular functional recovery or survival following valve replacement have been investigated, predictors for paravalvular aortic regurgitations after TAVR are less established. Postprocedural aortic regurgitation grade $2 / 4$ has been noted in about $12-20 \%$ of patients. In a recent study by Koos and colleagues [43], pre-TAVRCT scans were performed to calculate the Agastson calcium score and the routine echocardiographical protocol was used for pre and post-TAVR [44]. There was a correlation between very high Agastson scores (over 3000) and aortic insufficiency $3 / 4$ and higher. In a recent retrospective study, Sherif and colleagues [45] performed a univariate regression analysis on 50 successful CoreValve deployments and found that the LVOT-Ao angle, oval LVOT shape and prior AI all contributed significantly to the development of $3 / 4$ or $4 / 4$ aortic insufficiency. Three dimensional TEE is helpful in these cases as the geometry of the LV and the acuity of the differences in the LV axis and the LVOT-Ao axis, the shape of the LVOT and the amount of AI can all be assessed with 2D and 3D echocardiography. In a recent review of PARTNERI2-yearfollow-up data, perivalvular regurgitation has been associated withincreased mortality (Hazard ratio 2.11 for mild to moderate in severity). [46]

\subsection{Echocardiography during deployment $[47,48,49,50,51,52]$}

The imaging modalities that have described previously in this text for the pre-procedural evaluation of the aortic valve complex have been compared to the Hegar dilator technique in the 
work of Dashkevich and colleagues. Two dimensional TEE often underestimates the size of the aortic annulus when compared to CT that correlated well to the anatomical measurement. Whichever technique is used for sizing, the utility of live two and three dimensional echocardiography during the procedure is well accepted and outlined by Lerakis and colleagues.

Thefirststepin thedeploymentof theEdwards-Sapienvalveisachieving coaxiality of the delivery catheter with the LVOT-Aorta. This is best accomplished in the TEE probe in the high esophageal position, LVOT view, at 110-140 degrees of omniposition. Other authors have also recommended 3D TEE imaging with the use of "Zoom 3D" mode at 135, which allows a more precise positioning of the tip of the catheter and the prosthesis in relation to the aortic Annulus [53].

The next step is to align the aortic end of the THV with the tips of the native aortic valve leaflets. Fluoroscopy is used simultaneously and is discussed elsewhere [54]. After deployment, the 3dimensional transesophageal echocardiography short-axis view of the THV can be used to show a successful outcome with normal valve function and color Doppler can be used to assess possible valvular or perivalvular regurgitation. Mild to moderate perivalvular regurgitation is common after TAVR with the CoreValve. Sizing based on mean annulus diameter may help to reduce according to one recent retrospective review of 50 patients [55].

In clinical practice, optimal positioning of the CoreValve $5-10 \mathrm{~mm}$ proximal to the native aortic annulus occurs only $50 \%$ of the time by fluoroscopy. Non-optimal positioning has been found to have an inverse relationship to patient-prosthesis mismatch [56]. In our experience, the utility of TEE during deployment is greatest for recapturable valves such as the CoreValve as live evidence of perivalvular regurgitation can be useful before repositioning is performed. For Edwards-Sapien valves this is less crucial as the valve is deployed and cannot be recaptured. In very rare circumstances, the THV is deployed with the wrong orientation, thus producing severe, open valvular regurgitation. This can be assessed quickly with either $2 \mathrm{D}$ or $3 \mathrm{D}$ echo. Another valve is subsequently deployed within the inproperly deployed valve.

Positioning of the valve is critical as is the surveillance of the aortic valve complex and ascending aorta for any atheromas or loose calcium that can contribute to cerebrovascular accidents. There is no currently well-accepted description of the etiology of peri-procedural CVA.

Patient follow-up at three days post-procedure as well as months out is routine at our center. As with surgical aortic valve replacement, TTE is the imaging modality of choice for evaluation of prosthesis function during follow-up. Post-implantation gradients should be followed. There is flow acceleration at the valve stent as well as in the bioprosthetic valvular insertion point in the stent. This can lead to an overestimation of the trans-valvular gradients if the sample volume is placed inside the stent in the "subvalvular" position. As described above, the most proximal point of the stent should be used as the LVOT diameter and the sample volume should be placed there in the Doppler interrogation of the Edwards-Sapien valves [57]. Left ventricular hypertrophy should also be assessed. In fact, left ventricular hypertrophy that has been well described to be associated with clinically important aortic stenosis can be followed with transthoracic echocardiography and the Devereux formula in combination with the relative wall thickness has been used to evaluate the regression of increased ventricular wall thickness. 


\subsection{Limitations and alternative approaches}

\subsubsection{Cardiac computed tomography $[58,59,60]$}

As with other cardiac structures, the cardiologist and cardiac surgeon has multiple imaging modalities available for the evaluation of the aortic valve complex for TAVR planning. As described by Bloomfield and colleagues in a recent review paper, multi-detector CT, cardiac MRI and, of course, fluoroscopy can all be used to assess the area of interest. There are numerous advantages and disadvantages to each technique [61]. Fluoroscopy and CT are discussed elsewhere in this chapter. Although cardiac CT can provide a MFR image for multiple measurements, the most reproducible MDCT measurements of the annulus are the area-derived diameter and basal ring average diameter, with derived values generally larger than those obtained with echocardiography. More recently, the annular circumference has begun to emerge as the gold standard [62].

\subsubsection{Cardiac magnetic resonance imaging $[63,64]$}

Cardiac magnetic resonance allows for an anatomic and functional assessment of the aortic valve and aortic root. However, most CMR sequences are two-dimensional with the plane of imaging chosen at the time of the examination. Whole heart, echo-gated 3D cardiac MR can provide isotropic images for multiplanar reconstruction and shows the oval shape of the annulus with maximal and minimal diameters. Planimetric calculations can be made using steady-state free precession cine sequences. Limitations due to Gadollium contrast and long acquisition times are two reasons why it is less desirable and not routinely used in clinical practice.

\section{Future directions}

\subsection{Single cardiac cycle imaging [65]}

Although current limitations of 3D echo includes frame rate, acquisition volume and resolution there are both hardware and software advances coming in the future. Improvements in transducer design, parallel-processing and automated edge-detection and LV volume calculations will enable the sonographer to acquire higher quality images and the physician to answer clinical questions faster. Off-line processing will become simpler and less time consuming as well. Additional advances in the miniaturization of the probe and the echocardiogram machine itself will allow for wider use in the clinical setting.

\section{Conclusions}

Three dimensional echocardiography has emerged as a crucial imaging modality for TAVR planning and followup. With advanced post-processing techniques such as multi-plane 
reconstruction, I-slice and excellent temporal resolution of narrow-sector acquisition with the anatomical definition of wide-sector acquisition, the multi-disciplinary TAVR team is now able to perform this important procedure with great precision and with minimal risk.

\section{Author details}

Daniel Zalkind, Michael Kim and Ernesto E. Salcedo

*Address all correspondence to: ernesto.e.salcedo@ucdenver.edu

University of Colorado Hospital Anschutz Medical Campus, USA

\section{References}

[1] Leon, M. B, Smith, C. R, Mack, M, et al. Transcatheter aortic-valve implantation for aortic stenosis in patients who cannot undergo surgery. N Engl J Med (2010). , 363, 1597-607.

[2] Baumgartner, H, Hung, J, Bermejo, J, et al. Echocardiographic assessment of valve stenosis: EAE/ASE recommendations for clinical practice. J Am Soc Echocardiogr (2009). quiz 101-2., 22, 1-23.

[3] Blanke, P, Euringer, W, Baumann, T, et al. Combined assessment of aortic root anatomy and aortoiliac vasculature with dual-source CT as a screening tool in patients evaluated for ranscatheter aortic valve implantation. Am J Roentgenol (2010). , 195, 872-81.

[4] Blanke, P, Siepe, M, Reinohl, J, et al. Assessment of aortic annulus dimensions for Edwards SAPIEN Transapical Heart Valve implantation by computed tomography: calculating average diameter using a virtual ring method. Eur J Cardiothorac Surg (2010). , 38, 750-8.

[5] Delgado, V, Ng, A. C, Schuijf, J. D, et al. Automated assessment of the aortic root dimensions with multidetector row computed tomography. Ann Thorac Surg (2011). , 91, 716-23.

[6] Kurra, V, Kapadia, S. R, Tuzcu, E. M, et al. Pre-procedural imaging of aortic root orientation and dimensions: comparison between $\mathrm{X}$-ray angiographic planar imaging and 3-dimensional multidetector row computed tomography. JACC Cardiovasc Interv (2010). , 3, 105-13.

[7] Bagur, R, Rodes-cabau, J, Doyle, D, et al. Usefulness of TEE as the primary imaging echnique to guide transcatheter transapical aortic valve implantation. JACC Cardiovasc maging (2011). , 4, 115-24. 
[8] Anderson, R. H. Clinical anatomy of the aortic root. Heart (2000). , 84, 670-3.

[9] Ho, S. Y. Structure and anatomy of the aortic root. Eur J Echocardiogr (2009). i , 3-10.

[10] Piazza, N, De Jaegere, P, Schultz, C, Becker, A. E, Serruys, P. W, \& Anderson, R. H. Anatomy of the aortic valvar complex and its implications for transcatheter implantation of the aortic valve. Circ Cardiovasc Interv (2008). , 1, 74-81.

[11] Dashkevich, A, Blanke, P, Siepe, M, et al. Preoperative assessment of aortic annulus dimensions: comparison of noninvasive and intraoperative measurement. Ann Thorac Surg (2011). , 91, 709-14.

[12] Devereux, R. B, Alonso, D. R, \& Lutas, E. M. et. al. Echocardiographic assessment of left ventricular hypertrophy: comparison to necropsy findings. American Journal of Cardiology (1986). , 57, 450-8.

[13] Ibid. Baumgartner

[14] Veronesi, F, Corsi, C, Sugeng, L, et al. A study of functional anatomy of aortic-mitral valve coupling using 3D matrix transesophageal echocardiography. Circ Cardiovasc Imaging (2009). , 2, 24-31.

[15] Tseng, W, Meineri, M, \& Hahn, R. et. Al. Effect of transcatheter aortic valve replacement on the mitral valve. Moderated Poster Contribution. JACC (2012). s1): E , 1260-1260.

[16] Martin, A. G, Ruiz, J. M, \& Gonzalez, A. E. et. al. Multiplane Transesophageal Echocardiography in the Preoperative Evaluation of the Sinus of Valsalva Fistula to Right Chambers. Rev Esp Cardiol. (2002).

[17] Shanewise, J. S, Cheung, A. T, \& Aronson, S. et. al. ASE/SCA Guidelines for Performing a Comprehensive Intraoperative Multiplant Transesophageal Echocardiography Examination. JASE (1999). , 12, 884-900.

[18] Beraud, A. S, Schnittger, I, \& Miller, D. C. et. al. Multiplanar reconstruction of threedimensional transthoracic echocardiography improves the presurgical assessment of mitral prolapse. JASE (2009).

[19] Sutton, J. P. rd, Ho SY, Anderson RH. The forgotten interleaflet triangles: a review of the surgical anatomy of the aortic valve. Ann Thorac Surg (1995). , 59, 419-27.

[20] Roberts, W. C, Vowels, T. J, Ko, J. M, et al. Comparison of the structure of the aortic valve and ascending aorta in adults having aortic valve replacement for aortic stenosis versus for pure aortic regurgitation and resection of the ascending aorta for aneurysm. Circulation (2011). , 123, 896-903.

[21] Caselli, S, Mazzesi, G, Tritapepe, L, et al. D echocardiographic delineation of mitralaortic ntervalular fibrosa pseudoaneurysm caused by bicuspid aortic valve endocarditis. Echocardiography (2011). E , 1-4. 
[22] Frommelt, M. A, Snider, A. R, \& Bove, E. L. et. al. Echocardiographic assessment of subvalvular aortic stenosis before and after operation. JACC (1992). , 19, 1018-1023.

[23] Nagueh, S. E, Bierig, S. M, \& Budoff, M. J. et. al. American Society of Echocardiography Clinical Recommendations for Multimodality Cardiovascular Imaging of Patient with Hypertrophic Cardiomyopathy. JASE (2011). , 24, 473-98.

[24] Ibid. Bagur

[25] Shiotavon Ramm, et. al. Real-time Three-dimensional Echocardiography for Determining Right Ventricular Stroke Volume in an Animal Model of Chronic Right Ventricular Volume Overload. Circulation. (1998). , 97, 1897-1900.

[26] GopalKing, et. al. Left ventricular volume and endocardial surface area by three-dimensional echocardiography: comparison with two-dimensional echocardiography and nuclear magnetic resonance imaging in normal subjects. JACC(1993).

[27] Araiet. al. Accuracy of measurement LVEF by new real-time three-dimensional echocardiography...AJC(2004).

[28] Langet. al. Three-Dimensional Echocardiography: The Benefits of the Additional Dimension. JACC (2006).

[29] Goland, S, \& Trento, A. Iida Kiyoshi, et. al. Assessment of aortic stenosis by threedimensional echocardiography: an accurate and novel approach. Heart (2007). , 93, 801-807.

[30] Monaghan, M. J. Role of real time 3D echocardiography in evaluating the left ventricle. Heart (2006).

[31] Adegunsoye, A, Mundkur, M, Nanda, N. C, \& Hage, F. G. Echocardiographic evaluation of calcific aortic stenosis in the older adult. Echocardiography (2011). , 28, $117-29$

[32] Baumgartner, H, Hung, J, \& Bermejo, J. et. al. Echocardiographic assessment of valve stenosis: EAE/ASE recommendations for clinical practice. European Journal of Echocardiography (2009). , 10, 1-25.

[33] Carabello, B. A, \& Paulus, W. J. Aortic stenosis. Lancet (2009). , 373, 956-66.

[34] Ng, A. C, Delgado, V, \& Van Der Kley, F. et. al. Comparison of aortic root dimensions and geometries before and after transcatheter aortic valve implantation by 2and 3- dimensional tranesophageal echocardiography and multislice computed tomography. Circulation Cardiovascular Imaging (2010). , 3, 94-102.

[35] Ozkan, M, Gunduz, S, \& Yildiz, M. et. al. Diagnosis of the prosthetic heart valve pannus formation with real-time three-dimensional transoesophageal echocardiography. European Journal of Echocardiography (2010). E17 
[36] Clavel, M-A, Rodes-cabau, J, \& Dumont, E. et. al. Validation and characterization of transcatheter aortic valve effective orifice area measured by Doppler echocardiography. JACC Imaging (2011). , 4, 1053-62.

[37] Lancellotti, P, Tribouilloy, C, \& Hagendorgg, A. et. al. EAE recommendations for the assessment of valvular regurgitation. Part I: aortic and pulmonary regurgitation. European Journal of Echocardiography (2010). , 11(3), 223-244.

[38] Karalis, D. G, Bansal, R. C, Hauck, A. J, et al. Transesophageal echocardiographic recognition of subaortic complications in aortic valve endocarditis. Clinical and surgical implications. Circulation (1992). , 86, 353-62.

[39] Simpson, I. A, De Belder, M. A, \& Kenny, A. et. al. How to quantify valve regurgitation by echo Doppler techniques. British Heart Journal (1995). , 73, 1-9.

[40] Fang, L, Hsiung, M. C, \& Miller, A. P. et. al. Assessment of aortic regurgitation by live three0dimensional transthoracic echocardiographic measurements of vena contracta area: usefulness and validation. Echocardiography (2005). , 22, 775-81.

[41] Pouleur, A-C. le Polain de Waroux J-B, et. al. Accuracy of the flow convergence method for quantification of aortic regurgitation in patients with central versus eccentric jets. American Journal of Cardiology (2008). , 102, 475-80.

[42] Pate, G, \& Thomson, C. R. Munt Bl, et. al. Techniques for percutaneous closure of prosthetic paravalvular leaks. Catheter Cardiovascular Interventions. (2010). , 67, 158-66.

[43] Koos, R, Mahnken, A. H, \& Dohmen, G. et. al. Association of aortic valve calcification severity with the degree of aortic regurgitation after transcatheter aortic valve implantation. Int J Cardiol (2010). , 150, 142-5.

[44] Schultz, C, Rossi, A, Van Mieghem, N, \& Van Der Boon, R. et. al. Aortic annulus dimensions and leaflet calcification from contrast MSCT predict the need for balloon post-dilatation after TAVI with the Medtronic CoreValve prosthesis. Eurointevention, (2011). , 2011(7), 5-564.

[45] Sherif, M. A, Abdel-wahab, M, \& Stocker, B. et. al. Anatomic and procedural predictors of paravalvular aortic regurgitation after implantation of the Medtronic CoreValve bioprosthesis. JACC (2010). , 56, 1623-9.

[46] Kodali, S. K, Williams, M. R, \& Smith, C. R. Et. al. Two-Year Outcomes after Transcatheter or Surgical Aortic-Valve Replacement. NEJM. 366; , 18, 1686-1695.

[47] Ibid. Bagur

[48] Dashkevich, A, Blanke, P, Siepe, M, et al. Preoperative assessment of aortic annulus dimensions: comparison of noninvasive and intraoperative measurement. Ann Thorac Surg (2011). , 91, 709-14. 
[49] Rodes-cabau, J, Dumont, E, Boone, R. H, et al. Cerebral embolism following transcatheter aortic valve implantation: comparison of transfemoral and transapical approaches. J Am Coll Cardiol (2011). , 57, 18-28.

[50] Tzikas, A, Geleijnse, M. L, Van Mieghem, N. M, et al. Left ventricular mass regression one year after transcatheter aortic valve implantation. Ann Thorac Surg (2011). , 91, 685-91.

[51] Lerakis, S, Babaliaros, V. C, \& Block, P. C. et. al. Transesophageal echocardiography to help position and deploy a transcatheter heart valve. JACC Imaging (2010). , 3, 219-21.

[52] Filgueiras-rama, D, Lopez, T, \& Moreno-gomez, R. et. al. 3D transesophageal echocardiographic guidance and monitoring of percutaneous aortic valve replacement. Echocardiography (2010). , 27, 84-6.

[53] Jayasuriya, C, Moss, R. R, \& Munr, B. Transcatheter Aortic Valve Implantation in Aortic Stenosis: The Role of Echocardiography. JASE. (2011). , 24(1), 15-27.

[54] Durand, E, Borz, B, \& Godin, M. et. al. Transfemoral aortic valve replacement with the Edwards SAPIEN and Edwards SAPIEN XT prosthesis using exclusively local anesthesia and fluoroscopic guidance: feasibility and 30-day outcomes. JACC Cardiovascular Interventions. (2012). May; , 5(5), 461-7.

[55] Schultz, C. J, Tzikas, A, Moelker, A, \& Rossi, A. et. al. Correlates on MSCT of paravalvular aortic regurgitation after aortic valve implantation using the Medtronic COreValve prosthesis. Catheter Cardiovasc Interv, (2011). , 2011(78), 3-446.

[56] Ibid Jayasuriya

[57] Shames, S, Koczo, A, \& Hahn, R. et. al. In-stent flow acceleration in the SAPIEN trans-catheter aortic valve: impact on the echocardiographic assessment of valve function. JASE (2011).

[58] IbidBlanke, Delgado, Kurra

[59] Schultz, C. J, Molker, A, \& Piazza, N. et. al. Three dimensional evaluation of the aortic annulus using multislice computer tomography. European Heart Journal (2010). , 31, 849-856.

[60] Gurvitch, R, Webb, J, \& Yuan, R. et. al. Aortic annulus diameter determination by multidetector computed tomography: reproducibility, applicability and implications for transcatheter aortic valve implantation. JACC Intervention. (2011). , 4, 1235-45.

[61] Bloomfield, G. S, Gillam, L. D, \& Hahn, R. T. et. al. A Practical guide to multimodality imaging of transcatheter aortic valve replacement. JACC Imaging (2012). , 5, 441-455.

[62] Jilaihawi, H, Kashif, M, \& Fontana, G. et. al. Cross-Sectional Computed Tomographic Assessment Improves Accuracy of Aortic Annular Sizing for Transcatheter Aortic 
Valve Replacement and Reduces the Incidence of Paravalvular Aortic Regurgitation. JACC(2012).

[63] Ibid. Tadros.

[64] Burgstahler, C, Kunze, M, \& Loefller, C. et. al. Assessment of left ventricular outflow tract geometry in non-stenotic and stenotic aortic valves by cardiovascular magnetic resonance.Journal of Cardiovascular Magnetic Resonance (2006). , 8, 825-9.

[65] Lee, A. P, Lam, Y. Y, Yip, G. W, \& Lang, R. M. et. al. Role of real time three-dimensional transesophageal echocardiography in guidance of interventional proedures in cardiology. Heart (2010). , 96, 1485-1493. 